\title{
Windings of spherically symmetric random walks via Brownian embedding
}

\author{
Claude Bélisle \\ Department of Statistics, University of Michigan, Ann Arbor, MI 48109, USA
}

Received August 1989

Abstract: Let $X_{1}, X_{2}, X_{3}, \ldots$ be a sequence of i.i.d. $\mathbb{R}^{2}$-valued random variables with a spherically symmetric distribution. Let $\left(S_{n} ; n \geqslant 0\right)$ be its sequence of partial sums and let $(\phi(n) ; n \geqslant 0)$ be its winding sequence. Assuming only a mild moment condition we show, via Brownian embedding, that $2 \phi(n) / \log n$ converges in distribution to a standard hyperbolic secant distribution.

AMS 1980 Subject Classifications: Primary 60F05; Secondary 60J65, 60F15, 60F17.

Keywords: Random walks, Brownian motion, windings, asymptotic distribution.

\section{Introduction}

Let $X_{1}, X_{2}, X_{3}, \ldots$ be a sequence of i.i.d. $\mathbb{R}^{2}$-valucd random variables and let $S=\left(S_{n} ; n \geqslant 0\right)$ be its sequence of partial sums. In Bélisle (1989) we defined the winding sequence $(\phi(n) ; n \geqslant 0)$ of the random walk $S$ and we showed that if

(a) $X_{1}$ has mean vector zero and covariance matrix identity,

(b) there exists a finite constant $b$ such that $P\left[\left\|X_{1}\right\| \leqslant b\right]=1$, and

(c) either the distribution of $X_{1}$ is absolutely continuous with respect to Lebesgue measure in the plane, or the additive subgroup of $\mathbb{R}^{2}$ generated by the support of the distribution of $X_{1}$ is the lattice $\mathscr{L}_{d}=\left\{d z ; z \in \mathbb{R}^{2}\right\}$ for some $d>0$, then

$$
\frac{2 \phi(n)}{\log n} \stackrel{\mathrm{d}}{\rightarrow} W \quad \text { as } n \rightarrow \infty
$$

where $W$ is standard hyperbolic secant, i.e. $W$ has density $\frac{1}{2} \operatorname{sech}\left(\frac{1}{2} \pi w\right)$. (Condition (a) can be relaxed. Vector mean zero and nonsingular covariance matrix is enough. We hope to replace condition (b) by a moment condition. Condition (c) can be relaxed. Harris recurrence is enough.) The proof is long and it involves a rather complicated construction. The purpose of this note is to show that for spherically symmetric random walks satisfying a mild moment condition, the limit distribution result (1) follows easily, via Brownian embedding, from the analogous result for Brownian big windings.

\section{The result}

Let $F$ be a nondegenerate probability measure on $\mathbb{R}^{2}$ and assume that it satisfies the following conditions:

(i) $F$ is spherically symmetric (in the sense that for every rotation $Q$ on $\mathbb{R}^{2}$ and for every Borel set $B$ 
in $\left.\mathbb{P}^{2}, F(Q B)=F(B)\right)$.

(ii) $\quad \int_{\mathbb{R}^{2}}\|x\|^{2} \log ^{2}(\max \{1,\|x\|\}) F(\mathrm{~d} x)<\infty$.

Let $\mu$ be the probability measure on $\mathbb{R}_{+}$defined by

$$
\mu((a, b])=F\left(\left\{x \in \mathbb{R}^{2} ; a<\|x\| \leqslant b\right\}\right), \quad 0 \leqslant a<b<\infty .
$$

Let $B=(B(t): t \geqslant 0)$ be a standard 2-dimensional Brownian motion starting at the origin and let $R_{1}, R_{2}$, $R_{3}, \ldots$ be independent random variables with distribution $\mu$, independent of $B$. Let $\tau_{0}=0$ and for $j \geqslant 1$,

$$
\tau_{j}=\inf \left\{t \geqslant \tau_{j-1}:\left\|B(t)-B\left(\tau_{j-1}\right)\right\|=R_{j}\right\} .
$$

Finally, let

$$
X_{n}=B\left(\tau_{n}\right)-B\left(\tau_{n-1}\right)
$$

Observe that $X_{1}, X_{2}, X_{3}, \ldots$ is a sequence of i.i.d. $\mathbb{R}^{2}$-valued random variables with distribution $F$ and that its sequence of partial sums $S=\left(S_{n} ; n \geqslant 0\right)$ is the sequence $\left(B\left(\tau_{n}\right) ; n \geqslant 0\right)$. Furthermore the time increments $\tau_{j}-\tau_{j-1}, j \geqslant 1$, are i.i.d. This is our Brownian embedding representation of $S$. Observe also that $\left\|X_{j}\right\|=R_{j}$. From condition (ii) we have $\int_{\mathbb{R}^{2}}\|x\|^{2} F(\mathrm{~d} x)<\infty$ and since windings are invariant under scaling, there will be no loss of generality in assuming that $\int_{\mathbb{R}^{2}}\|x\|^{2} F(\mathrm{~d} x)=2$. (In view of condition (i) this is equivalent to the requirement that $F$ have covariance matrix identity). With this normalization we have $E\left[\tau_{j}-\tau_{j-1}\right]=1$. Now write

$$
\frac{2 \phi(n)}{\log n}=\frac{2 \theta_{B}(n)}{\log n}+\frac{2\left(\theta_{B}\left(\tau_{n}\right)-\theta_{B}(n)\right)}{\log n}+\frac{2\left(\phi(n)-\theta_{B}\left(\tau_{n}\right)\right)}{\log n}
$$

where $(\phi(n) ; n \geqslant 0)$ is the winding sequence of $S$ and where $\left(\theta_{B}(t) ; t \geqslant 0\right)$ is the Brownian big winding process

$$
\theta_{B}(t)=\int_{0}^{t} 1_{\{\|B(s)\|>1\}} \mathrm{d} \theta(s),
$$

as defined in Messulam and Yor (1982) and Pitman and Yor (1986). Below we prove the following two results:

\section{Proposition 1.}

$$
\theta_{B}\left(\tau_{n}\right)-\theta_{B}(n) \stackrel{\mathrm{P}}{\rightarrow} 0 \quad \text { as } n \rightarrow \infty .
$$

\section{Proposition 2.}

$$
\frac{\phi(n)-\theta_{B}\left(\tau_{n}\right)}{\log n} \stackrel{\mathrm{P}}{\rightarrow} 0 \quad \text { as } n \rightarrow \infty
$$

Now Messulam and Yor (1982) (see also Pitman and Yor, 1986) have shown that

$$
\frac{2 \theta_{B}(t)}{\log t} \stackrel{\mathrm{d}}{\rightarrow} W \text { as } t \rightarrow \infty
$$

where $W$ is standard hyperbolic secant. Thus we have: 
Theorem 1. For 2-dimensional random walks with increments having a distribution that satisfies conditions (i) and (ii), we have

$$
\frac{2 \phi(n)}{\log n} \stackrel{\mathrm{d}}{\rightarrow} W \text { as } n \rightarrow \infty
$$

where $W$ is standard hyperbolic secant.

\section{Proof of Proposition 1}

Fix $0<\varepsilon<1$. Choose $\delta_{\varepsilon}>0$ small enough so that

$$
P\left[\|B(1)\| \leqslant \frac{\delta_{\varepsilon}^{1 / 4}}{\sqrt{1-\delta_{\varepsilon}}}\right]<\frac{1}{4} \varepsilon \quad \text { and } \quad P\left[\sup _{0 \leqslant t \leqslant 1}\|B(t)\|>\frac{\sin \left(\frac{1}{2} \varepsilon\right)}{\sqrt{2} \delta_{\varepsilon}^{1 / 4}}\right]<\frac{1}{4} \varepsilon .
$$

Let $n_{\varepsilon}$ be large enough so that for all $n \geqslant n_{e}$,

$$
\boldsymbol{P}\left[\left|\frac{\tau_{n}}{n}-1\right|>\delta_{\varepsilon}\right]<\frac{1}{4} \varepsilon \quad \text { and } \quad \boldsymbol{P}\left[\|B(1)\| \leqslant \frac{1}{\sqrt{n\left(1-\delta_{\varepsilon}\right)}\left(1-\sin \left(\frac{1}{2} \varepsilon\right)\right)}\right]<\frac{1}{4} \varepsilon .
$$

Now observe that if we have $\left|\tau_{n}-n\right| \leqslant n \delta_{\varepsilon}$, if we have $\left\|B\left(n\left(1-\delta_{\varepsilon}\right)\right)\right\|\left(1-\sin \left(\frac{1}{2} \varepsilon\right)\right)>1$, and if we have $\sup _{n\left(1-\delta_{\varepsilon}\right) \leqslant t \leqslant n\left(1+\delta_{\varepsilon}\right)}\left\|B(t)-B\left(n\left(1-\delta_{\varepsilon}\right)\right)\right\| \leqslant\left\|B\left(n\left(1-\delta_{\varepsilon}\right)\right)\right\| \sin \left(\frac{1}{2} \varepsilon\right)$, then between time $n$ and time $\tau_{n}$ the Brownian path remains inside the disk of radius $\left\|B\left(n\left(1-\delta_{\varepsilon}\right)\right)\right\| \sin \left(\frac{1}{2} \varepsilon\right)$ centered at $B\left(n\left(1-\delta_{\varepsilon}\right)\right)$ and that disk does not intersect the unit disk centered at the origin. This implies that $\left|\theta_{B}(n)-\theta_{B}\left(\tau_{n}\right)\right| \leqslant \varepsilon$. Thus

$$
\begin{aligned}
\boldsymbol{P} & {\left[\left|\theta_{B}(n)-\theta_{B}\left(\tau_{n}\right)\right|>\varepsilon\right] } \\
\leqslant & P\left[\left|\tau_{n}-n\right|>n \delta_{\varepsilon}\right]+\boldsymbol{P}\left[\left\|B\left(n\left(1-\delta_{\varepsilon}\right)\right)\right\|\left(1-\sin \left(\frac{1}{2} \varepsilon\right)\right) \leqslant 1\right] \\
& +\boldsymbol{P}\left[\sup _{n\left(1-\delta_{\varepsilon}\right) \leqslant t \leqslant n\left(1+\delta_{\varepsilon}\right)}\left\|B(t)-B\left(n\left(1-\delta_{\varepsilon}\right)\right)\right\|>\left\|B\left(n\left(1-\delta_{\varepsilon}\right)\right)\right\| \sin \left(\frac{1}{2} \varepsilon\right)\right] \\
\leqslant & P\left[\left|\tau_{n}-n\right|>n \delta_{\varepsilon}\right]+\boldsymbol{P}\left[\left\|B\left(n\left(1-\delta_{\varepsilon}\right)\right)\right\|\left(1-\sin \left(\frac{1}{2} \varepsilon\right)\right) \leqslant 1\right] \\
& +\boldsymbol{P}\left[\sup _{n\left(1-\delta_{\varepsilon}\right) \leqslant t \leqslant n\left(1+\delta_{\varepsilon}\right)}\left\|B(t)-B\left(n\left(1-\delta_{\varepsilon}\right)\right)\right\|>\delta_{\varepsilon}^{1 / 4} n^{1 / 2} \sin \left(\frac{1}{2} \varepsilon\right)\right] \\
& +\boldsymbol{P}\left[\left\|B\left(n\left(1-\delta_{\varepsilon}\right)\right)\right\|<\delta_{\varepsilon}^{1 / 4} n^{1 / 2}\right] \\
= & P\left[\left|\frac{\tau_{n}}{n}-1\right|>\delta_{\varepsilon}\right]+\boldsymbol{P}\left[\|B(1)\|<\frac{1}{\sqrt{n\left(1-\delta_{\varepsilon}\right)}\left(1-\sin \left(\frac{1}{2} \varepsilon\right)\right)}\right] \\
& +\boldsymbol{P}\left[\sup _{0 \leqslant t \leqslant 1}\|B(t)\|>\frac{\sin \left(\frac{1}{2} \varepsilon\right)}{\sqrt{2} \delta_{\varepsilon}^{1 / 4}}\right]+\boldsymbol{P}\left[\|B(1)\| \leqslant \frac{\delta_{\varepsilon}^{1 / 4}}{\sqrt{1-\delta_{\varepsilon}}}\right]
\end{aligned}
$$

and we get

$$
P\left[\left|\theta_{B}(n)-\theta_{B}\left(\tau_{n}\right)\right|>\varepsilon\right]<\varepsilon \text { for all } n \geqslant n_{\varepsilon} .
$$

This proves the proposition. 


\section{Proof of Proposition 2}

Let $\lambda_{j}=(\phi(j)-\phi(j-1)), \eta_{j}=\theta_{R}\left(\tau_{j}\right)-\theta_{R}\left(\tau_{j-1}\right)$, and $\Delta_{j}=\lambda_{j}-\eta_{j}$. Then

$$
\phi(n)-\theta_{B}\left(\tau_{n}\right)=\sum_{j=1}^{n} \Delta_{j} .
$$

Below we show that $\boldsymbol{E}\left[\Delta_{j}^{2}\right]<\infty$ for $j \geqslant 1$ and $\boldsymbol{E}\left[\Delta_{j}^{2}\right]=\mathrm{O}(1 / j)$ as $j \rightarrow \infty$. By symmetry we have $\boldsymbol{E}\left[\Delta_{j_{1}} \Delta_{j_{2}}\right]=0$ for every $j_{1} \neq j_{2}$. Thus for every $\varepsilon>0$,

$$
\boldsymbol{P}\left[\left|\frac{\phi(n)-\theta_{B}\left(\tau_{n}\right)}{\log n}\right|>\varepsilon\right] \leqslant \frac{1}{\varepsilon^{2}(\log n)^{2}} \boldsymbol{E}\left[\left(\sum_{j=1}^{n} \Delta_{j}\right)^{2}\right]=\frac{1}{\varepsilon^{2}(\log n)^{2}} \sum_{j=1}^{n} \boldsymbol{E}\left[\Delta_{j}^{2}\right]=\mathrm{O}\left(\frac{1}{\log n}\right) .
$$

This proves the desired result. In order to obtain the desired bound on $E\left[\Delta_{j}^{2}\right]$, observe that if between time $\tau_{j-1}$ and time $\tau_{j}$ the Brownian path remains inside a disk that does not intersect the unit disk centered at the origin, then $\Delta_{j}=0$. Thus in particular

$$
\begin{aligned}
\boldsymbol{E}\left[\Delta_{j}^{2}\right]= & \int_{[0,1)} \int_{[0, \infty)} \boldsymbol{E}\left[\Delta_{j}^{2} \mid R_{j}=r,\left\|S_{j-1}\right\|=u\right] \mu(\mathrm{d} r) \mu^{(j-1)}(\mathrm{d} u) \\
& +\int_{[1, \infty)} \int_{[u-1, \infty)} \boldsymbol{E}\left[\Delta_{j}^{2} \mid R_{j}=r,\left\|S_{j-1}\right\|=u\right] \mu(\mathrm{d} r) \mu^{(j-1)}(\mathrm{d} u)
\end{aligned}
$$

where $\mu^{(j-1)}$ denotes the distribution of $\left\|S_{j-1}\right\|$. Now

$$
\begin{aligned}
\boldsymbol{E}\left[\Delta_{j}^{2} \mid R_{j}=r,\left\|S_{j-1}\right\|=u\right] & =\boldsymbol{E}\left[\left(\lambda_{j}-\eta_{j}\right)^{2} \mid R_{j}=r,\left\|S_{j-1}\right\|=u\right] \\
& \leqslant 3 \boldsymbol{E}\left[\lambda_{j}^{2} \mid R_{j}=r,\left\|S_{j-1}\right\|=u\right]+3 \boldsymbol{E}\left[\eta_{j}^{2} \mid R_{j}=r,\left\|S_{j-1}\right\|=u\right] .
\end{aligned}
$$

The first term is bounded above by $3 \pi^{2}$. The second term is equal to $3 E_{(u, 0)}\left[\theta_{B}^{2}(T((u, 0), r)]\right.$ and is bounded above by $3 E_{(0,0)}\left[\sup _{0 \leqslant t \leqslant T((0,0), u+r)} \theta_{B}^{2}(t)\right]$ where $E_{x}[\cdot]=E[\cdot \mid B(0)=x]$ and where $T(x, r)=$ $\inf \{t \geqslant 0:\|B(t)-x\|=r\}$. Now using conformal invariance (Pitman and Yor, 1986, Section 5) one gets

$$
\boldsymbol{E}_{(0,0)}\left[\sup _{0 \leqslant t \leqslant T((0,0), u+r)} \theta_{B}^{2}(t)\right] \leqslant c \log ^{2}(\max \{1, u+r\})
$$

for some finite constant $c$ (which may now change from line to line). Thus

$$
\begin{aligned}
\boldsymbol{E}\left[\Delta_{j}^{2}\right] \leqslant & \int_{[0,1)} \int_{[0, \infty)}\left(3 \pi^{2}+3 c \log ^{2}(\max \{1, u+r\})\right) \mu(\mathrm{d} r) \mu^{(j-1)}(\mathrm{d} u) \\
& +\int_{[1, \infty)} \int_{[u-1, \infty)}\left(2 \pi^{2}+3 c \log ^{2}(\max \{1, u+r\})\right) \mu(\mathrm{d} r) \mu^{(j-1)}(\mathrm{d} u) \\
\leqslant & \int_{[0,1)} \int_{[0, \infty)} \log ^{2}(\max \{1, r\}) \mu(\mathrm{d} r) \mu^{(j-1)}(\mathrm{d} u) \\
& +c \int_{[0, \infty)} \int_{[1, r+1]} \log ^{2}(\max \{1, r\}) \mu^{(j-1)}(\mathrm{d} u) \mu(\mathrm{d} r) \\
\leqslant & c \int_{[0, \infty)} \log ^{2}(\max \{1, r\}) \mu(\mathrm{d} r) \boldsymbol{P}\left[\left\|S_{j-1}\right\| \leqslant 1\right] \\
& +c \int_{[0, \infty)} \boldsymbol{P}\left[\left\|S_{j-1}\right\| \leqslant r+1\right] \log ^{2}(\max \{1, r\}) \mu(\mathrm{d} r) .
\end{aligned}
$$


Covering the disk of radius $\rho$ with $4 \rho^{2}$ unit squares and using Stone's theorem (Stone, 1970, Corollary 1) we get

$$
\boldsymbol{P}\left[\left\|S_{j}\right\| \leqslant \rho\right] \leqslant c \rho^{2} / j
$$

Thus

$$
\boldsymbol{E}\left[\Delta_{j}^{2}\right] \leqslant \frac{c}{j} \int_{[0, \infty)} r^{2} \log ^{2}(\max \{1, r\}) \mu(\mathrm{d} r)
$$

and condition (ii) yields the desired bound, i.e. $E\left[\Delta_{j}^{2}\right] \leqslant c / j$ for some finite constant $c$.

Remark 1. The case where $F$ is a uniform distribution over a circle centered at the origin was recently investigated by Berger (1987) and by Berger and Roberts (1988) using a totally different approach.

Remark 2. The bounds used in Section 4 are not sharp. It seems reasonable to hope that the condition $E\left[\left\|X_{1}\right\|^{2} \log ^{2}\left(\max \left\{1,\left\|X_{1}\right\|\right\}\right)\right]<\infty$ can be replaced by the weaker condition $E\left[\left\|X_{1}\right\|^{2}\right]<\infty$. It also seems reasonable to hope that this approach might work for general (not necessarily spherically symmetric) Brownian embeddable random walks. Spherical symmetry was used in the proof of Proposition 2 to obtain $E\left[\Delta_{j_{1}} \Delta_{j_{2}}\right]=0$ for all $j_{1} \neq j_{2}$. Without spherical symmetry, more care would be needed. This is reminiscent of the difficulties involved in handling the 'small windings' in Bélisle (1989).

Remark 3. Under the spherical symmetry assumption, the sequence $\Delta_{1}, \Delta_{2}, \Delta_{3}, \ldots$, is a martingale difference sequence and the computation of Section 4 suggests that $j \boldsymbol{E}\left[\Delta_{j}^{2}\right]$ converges to a finite positive constant. Thus it appears that the central limit theorem for martingales could be used to show that $\left(\phi(n)-\theta_{B}\left(\tau_{n}\right)\right) / \sqrt{\log n}$ is asymptotically normal.

\section{References}

Bélisle, C. (1989), Windings of random walks, Ann. Probab. 17(4).

Berger, M.A. (1987), The random walk winding number problem: convergence to a diffusion process with excluded area, J. Phys. A Math. Gen. 20, 5949-5960.

Berger, M.A. and P.H. Roberts (1988), On the winding number problem with finite steps, Adv. Appl. Probab. 20, 261-274.

Messulam, P. and M. Yor (1982), On D. Williams "pinching method" and some applications, J. London Math. Soc. (2) 26, 348-364.

Pitman, J.W. and M. Yor (1986), Asymptotic laws for planar Brownian motion, Ann. Probab. 14, 733-779.

Stone, C. (1967), On local and ratio limit theorems, Proc. Fifth Berkeley Symp. Math. Statist. Probab. (Univ. of California Press, Berkeley, CA). 